\title{
Effects of Alpha-Pinene Odor in Different Concentrations on Stress-Induced Hyperthermia in Rats
}

\author{
Hitomi AKUTSU'), Takefumi KIKUSUI ${ }^{1) *}$, Yukari TAKEUCHI ${ }^{1)}$ and Yuji MORI ${ }^{1)}$ \\ ${ }^{1)}$ Laboratory of Veterinary Ethology, The University of Tokyo, 1-1-1 Yayoi, Bunkyo-ku, Tokyo 113-8657, Japan
}

(Received 29 January 2003/Accepted 15 May 2003)

ABSTRACT. Stress-induced hyperthermia is observed in animals exposed to stressful conditions. In our previous study, plant-derived fragrances such as green odor and alpha-pinene were shown to suppress this stress response in rats. In the present study, we examined the concentration-dependence of the alpha-pinene effects on stress-induced hyperthermia. Male rats carrying telemetry transmitters were transferred individually to a new cage containing bedding that had been sprayed with $0.3,0.03$, and $0.003 \%$ concentrations of alphapinene or control solvent. Following transfer to the novel environment, the body temperature increased significantly, and this response was clearly suppressed when the cage was scattered with $0.03 \%$ alpha-pinene only. These results suggest that the effect of alpha-pinene on stress-induced hyperthermia can be observed only at a certain concentration.

KEY WORDS: alpha-pinene, concentration effect, hyperthermia.

J. Vet. Med. Sci. 65(9): 1023-1025, 2003

When an animal is exposed to stressful situations, autonomic responses including a temporary increase of body temperature, namely stress-induced hyperthermia or emotional fever, have been observed in several species [3, 4, 69, 24-26]. Recent studies have suggested that in phylogeny this stress response involving body temperature has emerged between amphibians and reptiles [5]. In our previous study, it was revealed that an external olfactory stimulation could affect this autonomic stress response. Namely, plant-derived fragrances such as green odor and alphapinene showed ameliorative effects on stress-induced hyperthermia [1]. In addition, by using this model Kikusui et al. could also show an opposite effect in which exposure to alarm pheromones resulted in an aggravation of this hyperthermia [13]. When the odorant concentration was changed, the impression or the effects accompanying that odor to the physiological response and behavior in vivo could be different. For example, the event-related potential P300 was altered its amplitude by emotional change, and the experiment using components of the green odor induced different amplitudes of P300 depending on its concentration [20]. Feeding behavior of goats was suppressed by only higher concentration of trans-2-hexenal [11]. These results suggest that each odorant has a certain concentration at which it is most effective. In the present study we therefore examined concentration-dependence of the effect of alphapinene on stress-induced hyperthermia by preparing both lower $(0.003 \%)$ and higher $(0.3 \%)$ concentrations in addition to the concentration $(0.03 \%)$ that in our previous study was confirmed to be effective.

The basic experimental procedures and animals were as described previously [1]. Briefly, male Wistar rats (9 weeks old: Clea Japan, Inc., Tokyo, Japan) weighing approxi-

\footnotetext{
* Correspondence to: Kikusui, T., Laboratory of Veterinary Ethology, The University of Tokyo, 1-1-1 Yayoi, Bunkyo-ku, Tokyo 113-8657, Japan.
}

mately 230-300 g were housed in groups of 4 animals per cage with wood shavings (Soft chip: Japan SLC, Inc., Sizuoka, Japan) as bedding. After being implanted with telemetry transmitters (TA11CTA-F40; Data Sciences International Co., St. Paul, MN), the animals were housed individually in cages containing bedding of paper chips (Paper clean: Japan SLC Inc., Sizuoka, Japan). Each cage was then placed in a soundproof box $(36 \times 54 \times 35 \mathrm{~cm}$ : width $\times$ depth $\times$ height; Muromachi Kikai, Co., Tokyo, Japan) that were kept on a 12 hr light-12 hr dark cycle (lights on from 0800 to $2000 \mathrm{hr}$ ) at constant room temperature $\left(21 \pm 2{ }^{\circ} \mathrm{C}\right)$, with food and water provided ad libitum. All the rats were experimentally naive and used only once in this study. They were habituated to the experimental procedures by handling $10 \mathrm{~min} /$ day for three days, and then divided randomly into three groups according to different alpha-pinene concentrations, i.e., $0.003 \%(\mathrm{n}=12), 0.03 \%$ $(n=11)$, and $0.3 \%(n=12)$. They were all exposed to a novel environment by being transferred into an unfamiliar cage containing new paper chips. Six animals from $0.3,0.03 \%$ odor group respectively, only five animals from the $0.03 \%$ group, were exposed to a solvent as the control. The alphapinene (Soda Aromatic Co., Ltd., Tokyo, Japan) was diluted with triethyl citrate freshly on the day before each experiment. Several minutes before the introduction of testing animals, $200 \mu \mathrm{l}$ of each alpha-pinene solution was scattered on the bedding of the cage. Behavioral responses were simultaneously recorded and later analyzed using an event recorder (CIF12, Toyo Sangyo, Toyama, Japan). Measured parameters were sniffing, rearing, grooming, resting and general activity. Data analyses were performed using StatView J-4.5 (Abacus Concepts, Berkeley, CA). Two-way analysis of variance (ANOVA) with repeated measures was used for comparison between each concentration and its control data, which was followed by Scheffe's F test as the post hoc analysis. The significance level was set at $\mathrm{p}=0.05$ 
for all statistical tests.

Figure 1 shows the time course changes in the body temperature in the male rats that were transferred to a new cage (novel environment). The transfer from the home cage to a new one resulted in an abrupt increase of body temperature, and the exposure to $0.03 \%$ alpha-pinene, but not $0.003 \%$ or $0.3 \%$, showed a decrease in the stress-induced hyperthermia $[0.003 \%: \mathrm{F}(1,660)=0.377, \mathrm{p}>0.9999 ; 0.03 \%$ : $\mathrm{F}(1,594)=1.863, \mathrm{p}<0.01 ; 0.3 \%: \mathrm{F}(1,660)=0.629, \mathrm{p}=0.9888]$. The heart rate was also increased by the transferral from home cage to novel environment, and exposure to $0.3 \%$ alpha-pinene diminished the increase of heart rate as compared to that when exposed to the solvent $[0.3 \%$ : $\mathrm{F}(1,140)=2.624, \mathrm{p}<0.01]$. Neither the exposure to $0.003 \%$ $[\mathrm{F}(1,140)=0.897, \mathrm{p}=0.5641]$ nor $0.03 \%[\mathrm{~F}(1,126)=1.380$, $\mathrm{p}=0.1722]$ alpha-pinene affected the heart rate change. Regarding the behavioral changes of rats during their exposure to a novel environment, the rats were active at the beginning of the test and then gradually grew sluggish. There was no difference with their own control in either the
$0.003 \%, 0.03 \%$ or $0.3 \%$ alpha-pinene exposed group.

In this study, we examined the effects of three different doses of alpha-pinene $(0.003 \%, 0.03 \%$, and $0.3 \%)$ in order to test the hypothesis that there exists a most effective concentration for alleviating stress response in rats. The obtained data suggest that an only $0.03 \%$ solution exerts a declining effect on stress-induced hyperthermia, which was in accordance with our previous findings [1]. Because of $0.03 \%$ alpha-pinene inhibited the stress-induced hyperthermia, and a higher dose $(0.3 \%)$ of alpha-pinene decreased cardiac response, it seems plausible that a single chemical odorant can be perceived via different neural pathways for expressing differing stress responses.

It appears possible that different concentrations of alphapinene bind to different olfactory receptors and thus induce differing neuronal activities. Each olfactory receptor cell expresses only one type of receptor gene out of more than $1000[17,19,27]$. Each olfactory receptor is considered to interact with plural odorants that share some chemical or structural characteristics; therefore, a single odorant can in

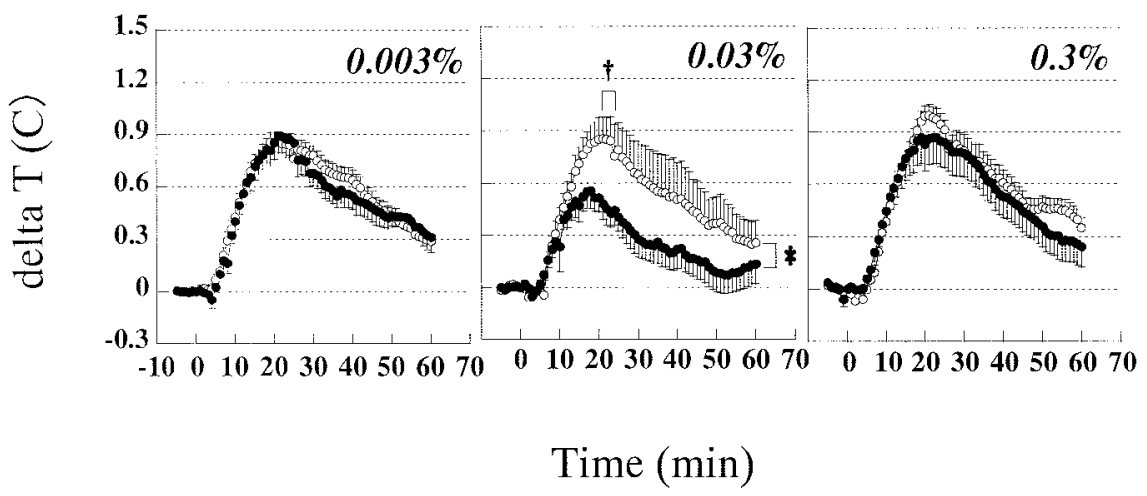

Fig. 1. Changes (means \pm SEM) in the body temperature of the male rats that were transferred from their home cage to a new cage where either the solvent (open circle) or one dilution of alpha-pinene (closed circle) had been scattered. Significant differences $(p<0.05)$ between exposures to the alpha-pinene and the solvent are shown as $*(\mathrm{p}<0.05$ with two-way ANOVA) or $\dagger$ ( $<<0.05$ with Scheffe's F).

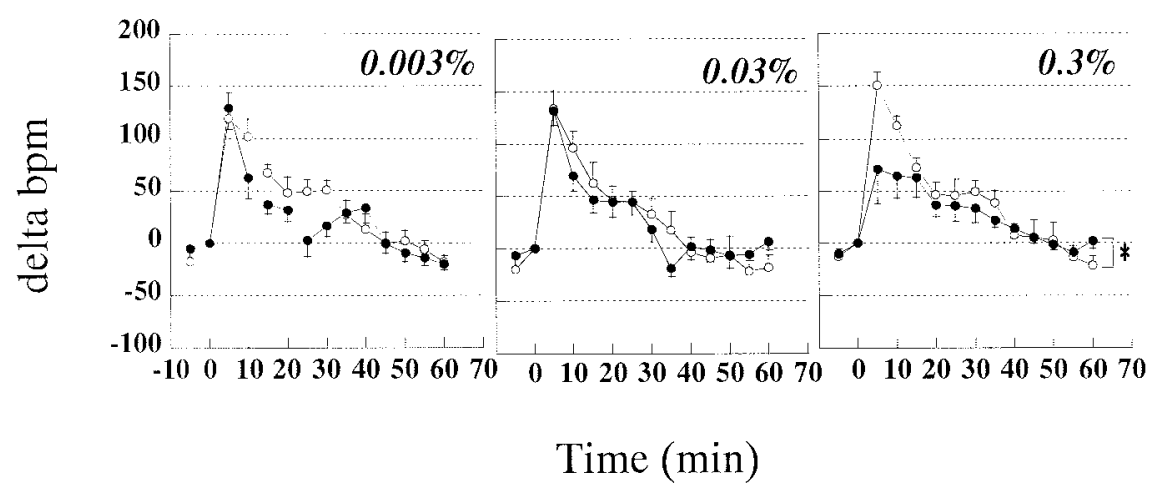

Fig. 2. Changes (means \pm SEM) in the heart rate of the male rats that were transferred from their home cage to a new cage where either the solvent (open circle) or one of the dilution[s] of alphapinene (closed circle) had been scattered. Significant differences $(\mathrm{p}<0.05)$ between exposures to the alpha-pinene and the solvent are shown as $*(\mathrm{p}<0.05$ with two-way ANOVA). 
turn be perceived by various types of olfactory receptors $[16,23]$. As each receptor does not have the same affinity to certain odorous substances, different concentrations of the same substance would activate various neuronal pathways differently [23]. Various endogenous factors such as hormones, cytokines, and neurotransmitters are supposed to be involved in the stress-induced hyperthermia [12, 15, 21, 22], and actually the administration of certain drugs (e.g., adrenaline-beta antagonists, 5-HT1A or 2A receptor antagonists, NOS inhibitors or prostaglandin synthesis blockers) have been reported to suppress this stress-induced hyperthermia $[2,10,14,18]$. Why the concentration-effect of alphapinene was exerted differently among autonomic parameters has not bee determined, though one possible explanation appears to be based on this hypothesis regarding differing thresholds among neural pathways to a given odor. In order to test this hypothesis, experimental approaches including a comparison between heart rate and body temperature responses to alpha-pinene would be interesting. Because these two autonomic parameters show different concentration effects, they could provide a useful model to further investigate the neurophysiological mechanism underlying these interesting biological phenomena.

We thank Dr. K.Sano (Soda Aromatic CO.) for providing odorant substances. This study was supported by the "Research for the Future" Program, The Japan Society for the Promotion of Science (JSPS-RFTF 97L00904), and by Grants-in-aid for Scientific Research from the Ministry of Education, Science, Sports and Culture (13306022) of Japan, and from CREST of the Japan Science and Technology Corporation.

\section{REFERENCES}

1. Akutsu, H., Kikusui, T., Takeuchi, Y., Sano, K., Hatanaka, A. and Mori, Y. 2002. Physiol. Behav. 75: 355-360.

2. Borsini, F., Brambilla, A., Grippa, N. and Pitsikas, N. 1999. Pharmacol. Biochem. Behav. 64: 137-146.

3. Borsini, F., Lecci, A., Volterra, G. and Meli, A. 1989. Psychopharmacology 98: 207-211.

4. Cabanac, A. and Briese, E. 1992. Physiol. Behav. 51: 95-98.

5. Cabanac, A. and Cabanac, M. 2000. Behav. Processes. 52: 89-
95.

6. Cabanac, A.J. and Guillemette, M. 2001. Physiol. Behav. 74: 475-479.

7. Cabanac, M. and Aizawa, S. 2000. Physiol. Behav. 69: 541545 .

8. Cabanac, M. and Bernieri, C. 2000. Behav. Processes. 49: 6168.

9. Cabanac, M. and Dardashti, M. 1999. Physiol. Behav .67: 347350.

10. De Paula, D., Steiner, A.A. and Branco, L.G. 2000. Physiol. Behav. 70: 505-511.

11. Dohi, H., Yamada, A., Entsu, S. and Fukukawa, T. 1997. Anim. Sci. Technol. 68: 474-480.

12. Groenink, L., van der Gugten, J., Zethof, T., van der Heyden, J. and Olivier, B. 1994. Physiol. Behav. 56: 747-749.

13. Kikusui, T., Takigami, S., Takeuchi, Y. and Mori, Y. 2001. Physiol. Behav. 72: 45-50.

14. Kluger, M.J., O'Reilly, B., Shope, T.R. and Vander, A.J. 1987. Physiol. Behav. 39: 763-766.

15. LeMay, L.G., Vander, A.J. and Kluger, M.J. 1990. Physiol. Behav. 47: 957-961.

16. Mori, K. 1995. Curr. Opin. Neurobiol. 5: 467-474

17. Mori, K. and Yoshihara, Y. 1995. Prog. Neurobio.l 45: 585619.

18. Nakamori, T., Morimoto, A., Yamaguchi, K., Watanabe, T., Long, N.C. and Murakami, N. 1993. Brain Res. 618: 155-159.

19. Ressler, K.J., Sullivan, S.L. and Buck, L.B. 1993. Cell 73: 597-609.

20. Sano, K., Tsuda, Y., Sugano, H., Aou, S. and Hatanaka, A. 2002. Chem. Senses. 27: 225-230.

21. Soszynski, D., Kozak, W., Conn, C.A., Rudolph, K. and Kluger, M.J. 1996. Neuroendocrinology 63: 459-467.

22. Soszynski, D., Kozak, W., Rudolph, K., Conn, C.A. and Kluger, M.J. 1997. Ann. N. Y. Acad. Sci. 813: 413-419.

23. Touhara, K., Sengoku, S., Inaki, K., Tsuboi, A., Hirono, J., Sato, T., Sakano, H. and Haga, T. 1999. Proc. Natl. Acad. Sci. U.S.A. 96: 4040-4045.

24. van den Buuse, M., Lambert, G., Fluttert, M. and Eikelis, N. 2001. Behav. Brain. Res. 119: 131-142.

25. van den Buuse, M., Van Acker, S.A., Fluttert, M. and De Kloet, E.R. 2001. Psychophysiology 38: 490-499.

26. van den Buuse, M., van Acker, S.A., Fluttert, M.F. and de Kloet, E.R. 2002. Physiol. Behav. 75: 207-215.

27. Vassar, R., Ngai, J. and Axel, R. 1993. Cell 74: 309-318. 